Communications in Physics, Vol. 26, No. 4 (2016), pp. 381-392

DOI:10.15625/0868-3166/26/4/8805

\title{
DEPENDENCE OF MELTING PROCESS ON SIZE AND EDGE TYPE OF GRAPHENE NANORIBBONS
}

\author{
NGUYEN THI THUY HANG ${ }^{\dagger}$ \\ Ho Chi Minh city University of Technology, \\ Vietnam National University - Ho Chi Minh City, \\ 268 Ly Thuong Kiet Street, ward 14, District 10, Ho Chi Minh City, Vietnam \\ ${ }^{\dagger}$ E-mail: hangbk@hcmut.edu.vn \\ Received 23 October 2016 \\ Accepted for publication 17 February 2017
}

\begin{abstract}
The study of variation of the size, armchair and zigzag type effects on the melting process of graphene nanoribbons (GNR). A numerical thermodynamical model has been devoted for the study. The phase transition has first order behaviour. The formation of different defects, ring size and coordination number is dependent on the size and the edge type of GNR. The nuclei of heating appear at temperature around $2300 \mathrm{~K}$ and that can be considered as pre-melting point. The melting process shows the case that the results of Berezinsky-Kosterlitz-Thouless-NelsonHalperin-Young theory cannot be applied.
\end{abstract}

Keywords: graphene nanoribbon, armchair, zigzag, size dependence, defects, phase transition.

Classification numbers: 61.48.Gh, 61.46.-w, 81.05.ue.

\section{INTRODUCTION}

As is know that graphene has been intensively studied due to its peculiar physical properties [1,2] and its potential applications for modern electronic devices [3-5]. However, graphene is zero-gap where the conduction and valence bands touch each other at a point called the Dirac point but do not overlap each other and this attribute makes a big problem for digital electronics [6]. Graphene nanoribbons (GNR) is one of the best solutions on this problem [7,8]. It has been reported that GNR can be considered as a material with controllable band gap, depending on the edge structure and ribbon width [7-9]. It has also been suggested that zigzag type of GNR (ZGNR) may be considered as a perspective material for spintronics because it shows a half metal 
properties when an external field is applied [8]. Note that the electronic structures of armchair type of GNR (AGNR) are presented using density functional theory [8]. The results indicate that AGNR is semiconductor which the band gap depends on the ribbon width. The $0.5 \mathrm{eV}$ band gap for the 2.5nm width of AGNR is reported [10]. There is no magnetic field in AGNR while ZGNR is predicted to have a magnetic insulating ground state with ferromagnetic ordering at each zigzag edge and antiparallel spin orientation between the two edges.

There are many studies on the measurements of the melting point of graphite ( [11] and related references). The melting temperature of graphite has been studied experimentally at pressures around $10 \mathrm{GPa}$ and the results present a large spread between $4000 \mathrm{~K}$ and $5000 \mathrm{~K}$ [11]. With the long-range carbon bond-order potential by Los and Fasolino (LCBOP II) [12], free energy calculations give the melting point at $4250 \mathrm{~K}$, almost independent of pressure between 1 and $20 \mathrm{GPa}$ [12]. At zero pressure, however, graphite sublimates before melting at $3000 \mathrm{~K} \mathrm{[11].}$ Monte Carlo simulations with LCBOP II at zero pressure show that, at $3000 \mathrm{~K}$, graphite sublimates through detachment of the graphene layers [12].

One layer of graphite - graphene then is studied [13]. The melting point is estimated from the two-dimensional Lindemann criterion and from extrapolation of simulation for different heating rates is around $4900 \mathrm{~K}$. However, there is only a couple of studies on the GNR. Lee et al. carried out tight-binding molecular dynamics simulations using model with 120 atoms and indicated that different types of edge of GNRs (i.e., zigzag and armchair) affect on the melting process in various ways [14]. They found that at $2800 \mathrm{~K}$ reconstruction process of two hexagons into a pentagon-heptagon occurs at the edge of a ZGNR. Because of the high structural stability of these systems [15], it is difficult to obtain the melting temperature and information on the equilibrium phases at temperatures close to and exceeding the melting point of GNR, experimentally. Therefore, molecular dynamics simulation of melting GNR will give the information in melting process.

In this study, we performed a detailed molecular dynamics study of the melting of GNR with various sizes of model as well as different edges of structure. The meting process is studied via total energy per atom, occurrence/growth of various types of defects upon heating, the change in coordination number and the formation of different ring sizes. Details about the calculations are presented in Sec. II. Results and discussions related to the affection of size and edge type on melting process can be found in Sec. III. Conclusions are given in the last section of the paper.

\section{CALCULATION}

There are some empirical potentials for covalent materials which are designed to give a good description close to equilibrium. In the literature we can distinguish various types of models, namely, Stillinger-Weber (SW) type potentials [16-19], Tersoff type bond order potentials (BOPs) [20-23], embedded atom models (EAMs) [24,25], higher order bond order potentials derived from tight binding models (TBBOPs) [26,27] and reactive bond order (REBO) potential [21]. However, for carbon which is smaller than silicon, the above empirical potentials are insufficient to describe the equilibrium honeycomb structure due to the stronger coupling between the free orbitals of under coordinated neighboring atoms which results in a stronger p-bond formation and can lead to complicated hybridization situations. In particular, the intrinsic long-range bond order potential for carbon (LCBOP I) [28] smoothly bridges the gap between the strong covalent and the weak intermolecular interactions, important for bond breaking and formation. The LCBOP I is an appropriately parametrized mix of a short-range Brenner-like bond order potential and a long-range, 
radial potential. Besides accurate values for bond distances, binding energies and stretching force constants for a large set of coordination environments, it gives good elastic constants for diamond and graphite, a reasonable description of the reaction path for the bulk diamond to graphite transition, as well as a good description of the interlayer interaction energy in graphite over a range of interlayer distances, as compared to experimental and/or ab initio data [29,30]. The total binding energy $\mathrm{Eb}$, according to the LCBOP I, is written as a sum of pair terms:

$$
E_{b}=\frac{1}{2} \sum_{i, j}^{N} V_{i j}^{t o t}=\frac{1}{2} \sum_{i, j}^{N}\left(f_{c, i j} V_{i j}^{S R}+S_{i j} V_{i j}^{L R}\right),
$$

where the total pair interaction $V_{i j}^{t o t}$ is the sum of a short-range part, $f_{c, i j} V_{i j}^{S R}$ describing the covalent interaction, and a long-range part $S_{i j} V_{i j}^{L R}$. The function $f_{c, i j}=f_{c, i j}\left(r_{j j}\right)$ is a smooth cutoff function, and $S_{i j}$ is a switching function, to exclude first neighbors, given by:

$$
S_{i j}=1-f_{c, i j} .
$$

The short-range part is written as:

$$
V_{i j}^{S R}=V_{R}\left(r_{i j}\right)-B_{i j} V_{A}\left(r_{i j}\right),
$$

where $V_{R}$ and $V_{A}$ are repulsive and attractive radial pair potentials respectively and, $B_{i j}$ is the bond order, containing many body effects.

In order to perform the calculations we use the software package Large-Scale Atomic/Molecular Massively Parallel Simulator (LAMMPS), designed to solve various problems by the methods of classical molecular dynamics [31].

In this work, three models of ZGNR with 5000, 10000 and 20000 atoms are presented to study the size dependence of melting process. Additionally, two more models of AGNR with 5000 and 10000 atoms are presented to study the dependence on edge types (AGNR and ZGNR) of melting process. Note that, the initial model has armchair structure along the $x$ Cartesian coordinates and zigzag structure along the $y$ one. Periodic boundary conditions (PBCs) are applied only along the $x$ Cartesian coordinate while non-periodic boundary with elastic reflection behaviour is along the y Cartesian coordinate in order to get AGNR. To get ZGNR, PBCs is applied along the y Cartesian coordinate while non-periodic boundary with elastic reflection behaviour is along the $x$ Cartesian coordinate.

Our simulation scenario for each model includes three stages.

Stage 1: The initial crystalline models under PBCs have been relaxed in the isothermalisobaric ensemble (NPT ensemble) for $3 \times 10^{5} \mathrm{MD}$ steps at $T_{0}=300 \mathrm{~K}$. Note that $1 \mathrm{MD}$ step takes 0.0002 picoseconds.

Stage 2: Before applying the non-periodic boundary with elastic reflection behaviour along the respectively Cartesian coordinates to get the desired edge type of GNR (i.e AGNR and ZGNR), an empty space of $20 \AA$ is added. Then, the system is left to equilibrium further for $2 \times 10^{5} \mathrm{MD}$ steps at $T_{0}=300 \mathrm{~K}$ using NVT ensemble simulation corresponding to the new boundaries of the simulation cell.

Stage 3: A temperature $T_{i}\left(T_{i}=12000 \mathrm{~K}\right)$ is chosen. It is higher than the melting point of graphene or graphite to guarantee that we can get all information on the phase transitions, intermediate structures at temperatures close to and exceeding the melting point of AGNR and 
ZGNR upon heating. The system is heated from the temperature $T_{0}$ to $T_{i}$ in the NVT ensemble. The heating rate is $5 \times 10^{11} \mathrm{~K} / \mathrm{s}$.

We employ the Interactive Structrue Analysis of Amorphous and Crystalline Systems (ISAACS) software for calculating the ring statistics [32]. We use the Visual Molecular Dynamics (VMD) software (Illinois Univ.) for 2D visualization of atomic configurations [33]. For calculations of the rings, the 'shortest path' rule is applied [31]. Temperature of the system is increased linearly via velocity rescaling as follows: $T=T_{0}+\gamma t$. Here, $T_{0}=300 \mathrm{~K}$ and $\gamma$ is a heating rate, $t$ is a time required for heating. Models, obtained at each temperature have been relaxed at a given temperature for $6 \times 10^{5} \mathrm{MD}$ steps before analyzing the structural characteristics or $2 \mathrm{D}$ visualization.

\section{RESULTS AND DISCUSSION}

The total energy is presented to study the melting process of AGNR and ZGNR (Fig. 1). The model has the square sharp with 10000 atoms. One can see that, for both AGNR and ZGNR, the total energy can be considered through three ranges of temperature:

i) Range 1 (from $300 \mathrm{~K}$ to $6900 \mathrm{~K}$ ): The total energy linearly increases upon heating indicating that the models remain in solid state. So that vibrational motion of atoms near their equilibrium positions dominates in the model. However, there is a sign on the total energy which shows a bit vibration from the linear law at quite low temperature - around $2300 \mathrm{~K}$ (the dash circle in Fig. 1). One can see that this change of the total energy is caused by some types of defects which formed at temperature around $2300 \mathrm{~K}$ but this change of total energy is not large enough to be able to massive collapse of model. Details on the defects upon heating are presented below.

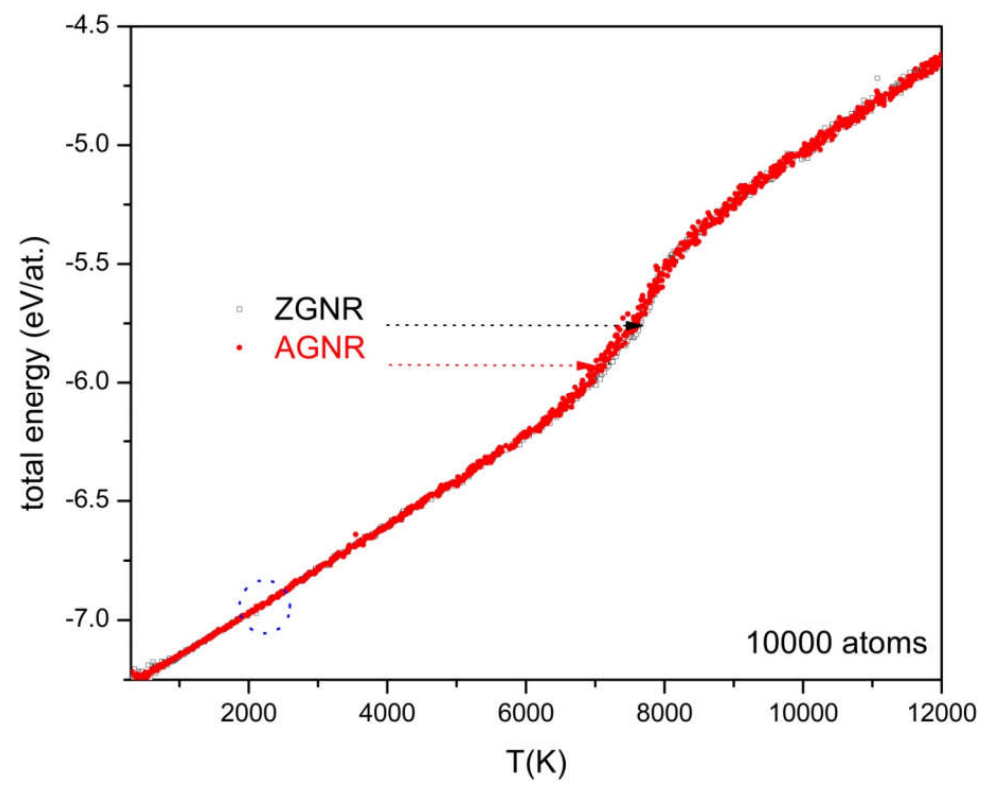

Fig. 1. Temperature dependence of total energy per atom: 10000 atoms, square sharp, square dot - ZGNR, circle dot - AGNR. 
ii) Range 2 (from $6900 \mathrm{~K}$ to $8000 \mathrm{~K}$ ): The contribution of anharmonic motion of atoms is strong enough leading to massive collapse of a crystalline matrix and the total energy starts to deviate clearly from the linear law. The total energy has a sharp increase exhibiting a first orderlike phase transition (Fig. 1).

iii) Range 3 (upon $8000 \mathrm{~K}$ ): The total energy increases linearly with temperature relating to the liquid state (Fig. 1).

One can see that in the case of the same number atom (10000 atoms) and edge length (square dot), the behaviour of total energy has a bit difference between AGNR and ZGNR in the first range of temperature: the total energy of AGNR (circle dot in Fig. 1) seems to deviate from the linear law sooner than the one of ZGNR (square dot in Fig. 1). The statistical noise of AGNR is more complex in comparison with ZGNR (circle dot in Fig. 1).

To have an overview about the affection of edge structure on the melting process, we present one more case for AGNR and ZGNR with the same number atom (5000 atoms) and different edge lengths (rectangle sharp). Detail about the rectangle sharp as follow: i) To get ZGNR, the 161- $\AA$ armchair edge (x Cartesian direction) is fixed while the $81-\AA \AA$ zigzag edge (y Cartesian direction) is put under the periodic condition; ii) To get AGNR, the $81-\AA ̊$ armchair edge (x Cartesian direction) is fixed while the 161- $\AA$ zigzag edge (y Cartesian direction) is put under the periodic condition.

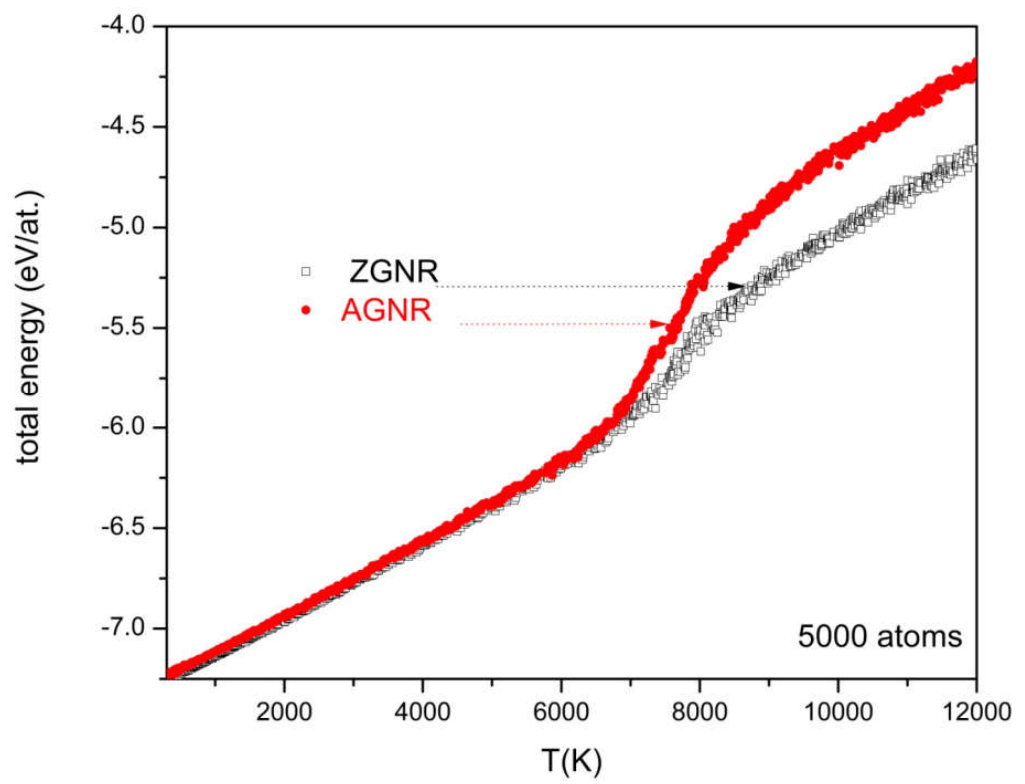

Fig. 2. Temperature dependence of total energy per atom: 5000 atoms, rectangle sharp, square dot - ZGNR, circle dot - AGNR.

One can see that the total energy of AGNR (circle dot in Fig. 2) is a bit higher than the one of ZGNR (square dot in Fig. 2) in the whole range of studied temperature. The total energy of AGNR deviates from the linear law at temperature about $6000 \mathrm{~K}$ and it is quite lower than the one of ZGNR which can clearly observe in Fig. 2. From temperature around $6000 \mathrm{~K}$ to the end of the melting process, the behaviour of total energy of AGNR is absolutely higher from ZGNR 
(Fig. 2). As one can see that the total energy of AGNR has tendency to shift to the higher region with increasing temperature in comparison with the one of ZGNR. From this point of view, it can be seen that there is a difference in binding energy between armchair and zigzag types (Figs. 1,2).

This is because at armchair edges neighbouring dangling bonds can pair-up to form $\mathrm{C} \equiv \mathrm{C}$ triple bonds that constitute a considerable stabilization effect compared to the isolated dangling bonds at zigzag edges.

To study the size dependence of melting process, three models of ZGNR with different numbers of atoms are presented: 5000, 10000 and 20000 atoms. The total energy per atom is studied (Fig. 3). The behaviours of models with 5000 atoms (square dot in Fig. 3) and 10000 atoms (circle dot in Fig. 3) are almost the same. When the number of atom increases to 20000 atoms (triangle dot in Fig. 3), the total energy decreases clearly in comparison with the other models. One can see that, in the range of this study, the increasing number of atoms in the model leads to decrease statistical noise and total energy per atom upon heating.

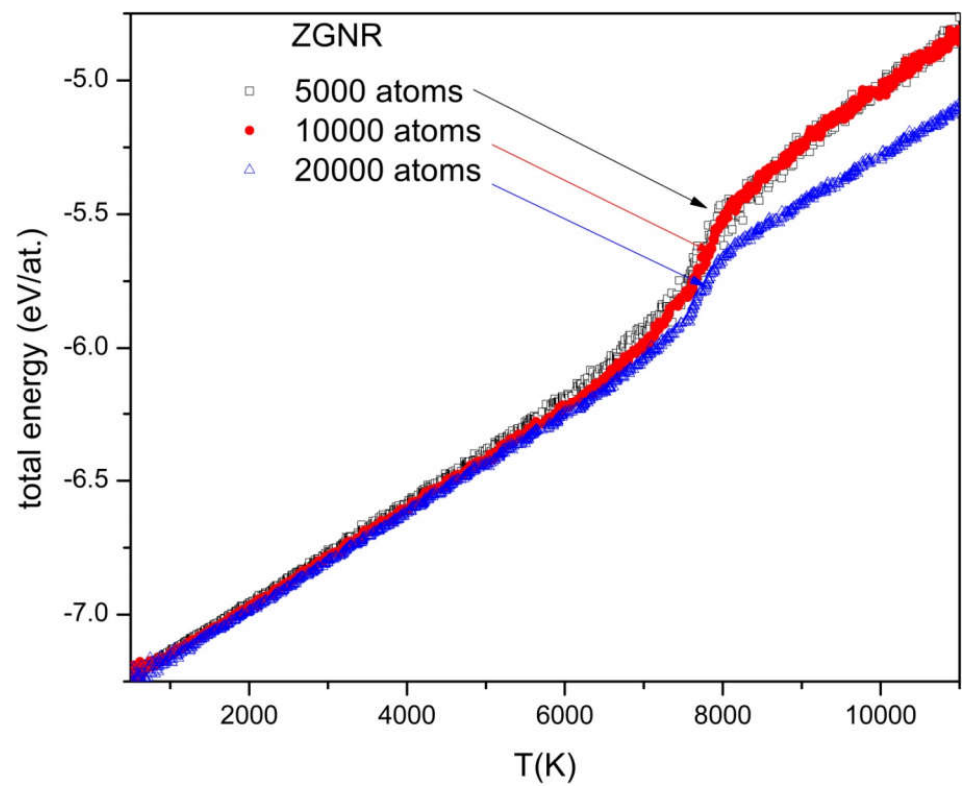

Fig. 3. Temperature dependence of total energy per atom for different sizes of ZGNR: 20000 atoms triangle dot; 10000 atoms circle dot; 5000 atoms square dot.

The mechanism of melting process can be considered as follow: During heating process, the atoms vibrate around their equilibrium positions in the system. At high temperature, the contribution of anharmonic motion of atoms is strong enough to distort the solid structures. The valence bonds of $\mathrm{C}-\mathrm{C}$ atoms then are broken causing the formation of structural defects, different ring sizes and chains. This process can be observed via distributions of coordination number, ring distributions or structural defects at various temperatures. In this work, AGNR and ZGNR with 10000 atoms (square sharp) are presented to study the affection of edge structures on the forming of coordination number, ring distributions and structural defects. 
Temperature dependence of the fraction of atoms with various coordination numbers can be seen in Fig. 4.

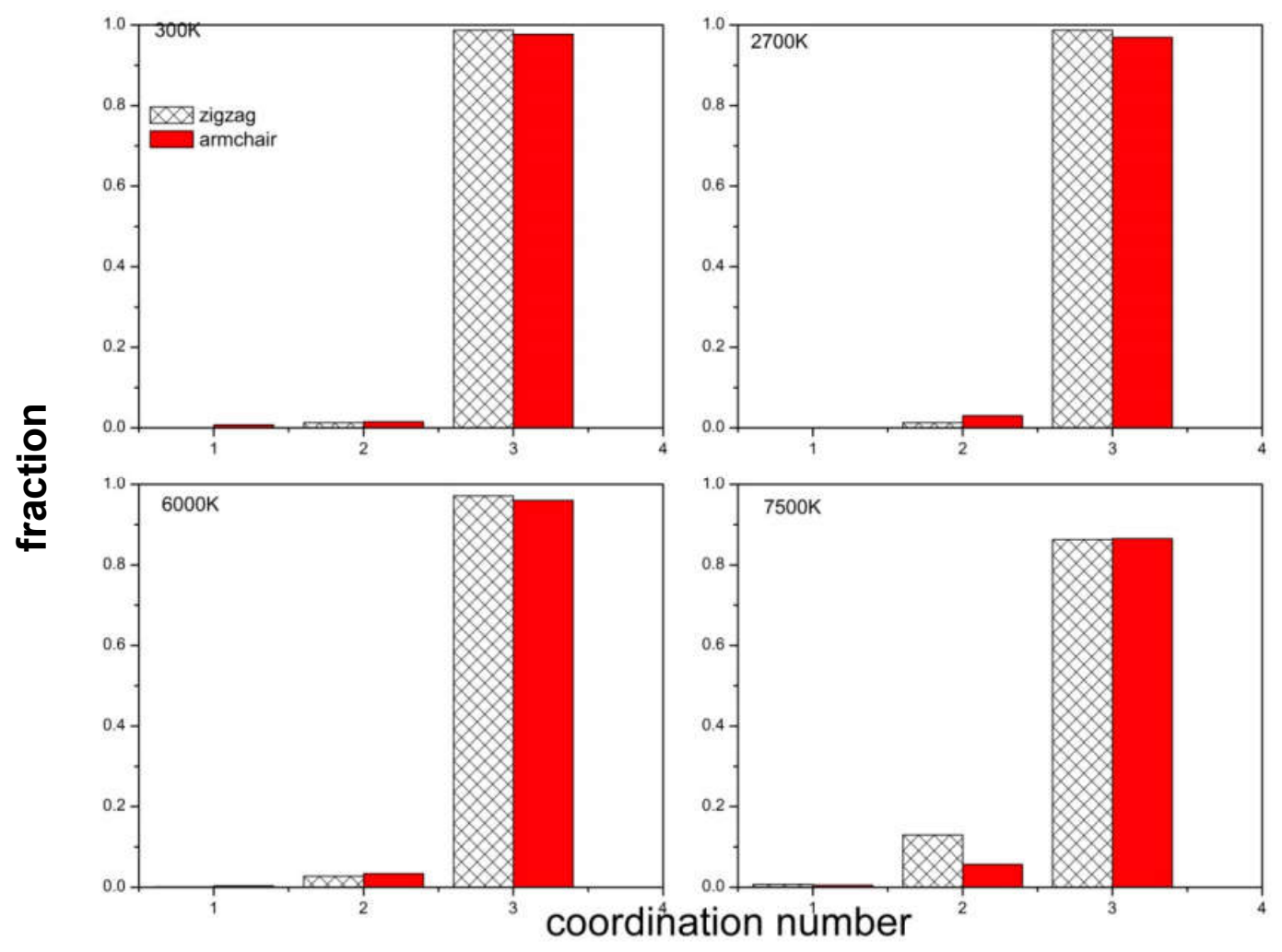

Fig. 4. Coordination number distributions at various temperatures: check column ZGNR, solid column - AGNR.

One can see that atoms with $Z \neq 3$ can be considered as structural defects and we find no atoms with $Z>3$ in the whole temperature range in this work for both AGNR and ZGNR indicating that there is no overcoordinated - defect in 2D space. Note that the evolution of atoms with $Z=2$ is related to the formation and breaking of rings and the formation of dangling bonds, respectively. Here we presented the coordination number at different temperatures (Fig. 4). One can see that the evolution of atoms with $Z=2$ of AGNR are higher than that of ZGNR in the range of temperature lower than $6000 \mathrm{~K}$ (Figs. 4a,b). At temperature $6000 \mathrm{~K}$, atoms with coordination number $Z=2$ of ZGNR increase fast in comparison with AGNR and higher than that of AGNR at temperature $8000 \mathrm{~K}$ (Figs. 4c,d). As it is analysed for the total energy that at temperature upon $8000 \mathrm{~K}$ (range 3 ) the models are on the liquid state, however, the atoms with coordination number $Z=2$ are still high enough indicating that models still remain solid atoms.

The formation of rings can be seen via temperature dependence of the ring distributions for both AGNR and ZGNR (Fig. 5).

Mean ring size remains equaled to 6 at temperature $300 \mathrm{~K}$ indicates that models remain in solid state with honeycomb structure (Fig. 5a) before changing upon heating due to the formation 


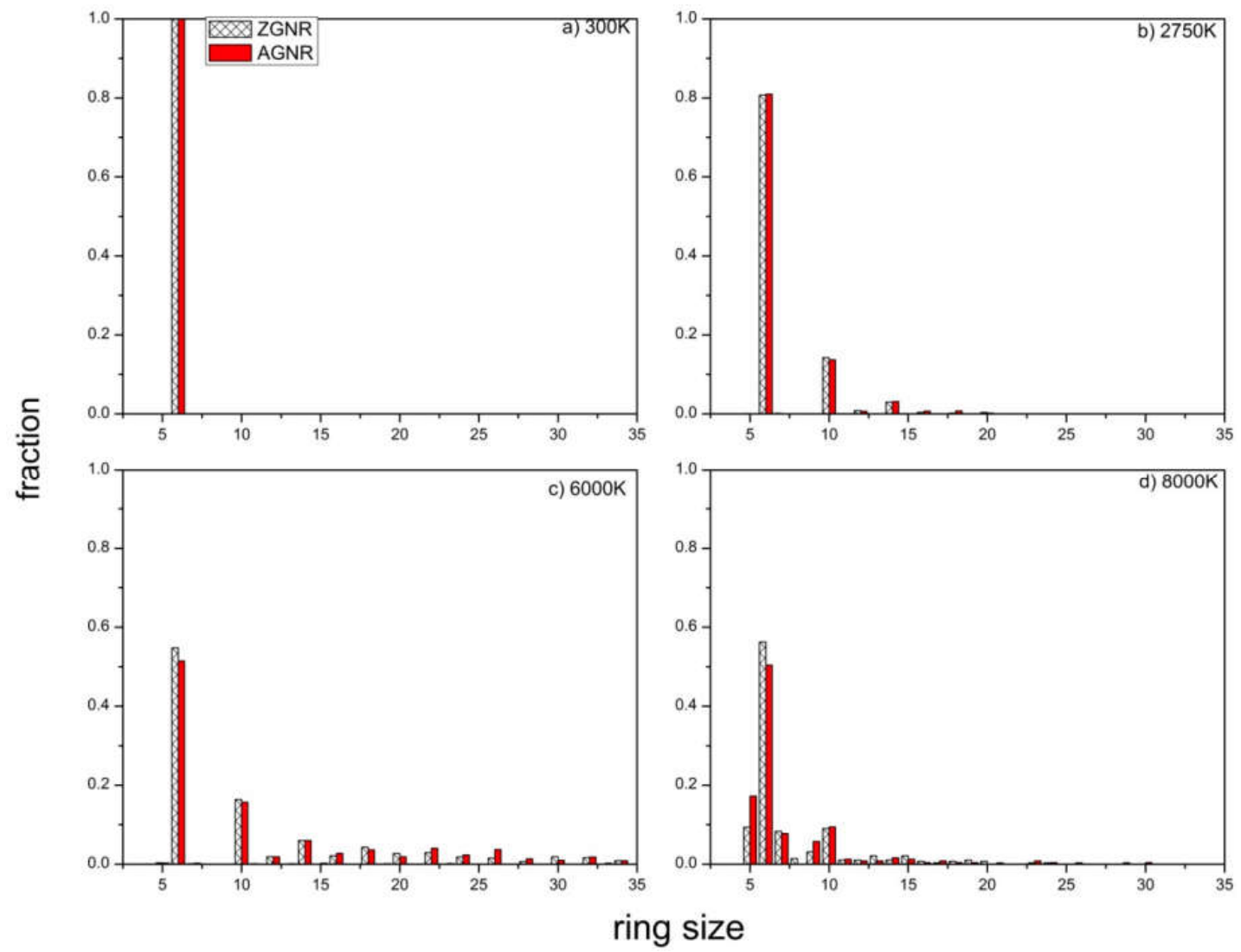

Fig. 5. Ring distributions at various temperatures: check column - ZGNR, solid column - AGNR.

of different defects. One can see that at temperature around $2780 \mathrm{~K}$, fraction of 5-fold, 7-fold and 10-fold rings increases indicating that the nuclei of heating appear at quite low temperature (Fig. 5b). At temperature $6000 \mathrm{~K}$, the rings with various sizes ranged from 9-fold to 34-fold ones are formed (Fig. 5c). In the range of temperature from $300 \mathrm{~K}$ to $6000 \mathrm{~K}$, the formation of rings of AGNR is more complex than that of ZGNR (Fig. 5a-c). In contrast, at temperature $8000 \mathrm{~K}$, the formation of defects of ZGNR is more active than that of AGNR, fraction of large rings decreases while fraction of small rings (smaller than 6 fold one) increases indicating a breaking of large rings to form chain (Fig. 5d). However, at $8000 \mathrm{~K}$ the fraction of 6 -fold ring of both ZGNR and AGNR decreases upon heating but it is still high (50\% and 57\% for AGNR and ZGNR, respectively) indicating that the models still remain the honeycomb structure.

One can see that in the range of temperature upon $8000 \mathrm{~K}$ where the liquid state takes place, the models still remain solid atoms (the 6 fold ring as well as atoms with coordination number $Z=3$ ) indicating that the melting process of both ZGNR and AGNR is not supported by Berezinsky-Kosterlitz-Thouless-Nelson-Halperin-Young (BKTNHY) theory [34-36]. Based 
on BKTHNY theory, melting of $2 \mathrm{D}$ crystals occurs via unbinding of topological defects. However, the validity of the BKTHNY theory has been under debate since results of simulations and experiments do not agree with each other (see [37-44] and references therein). In particular, the 5-7 dislocations as well as Stone-Wales (SW) defects, and vacancies (DVs) are found at the edges of GNR. This type of defects has been found by both experiment and theory $[45,46]$ and their subsequent discussion is different from BKTHNY theory. Details of this can be seen via a side view of 2D visualization in Fig. 6.

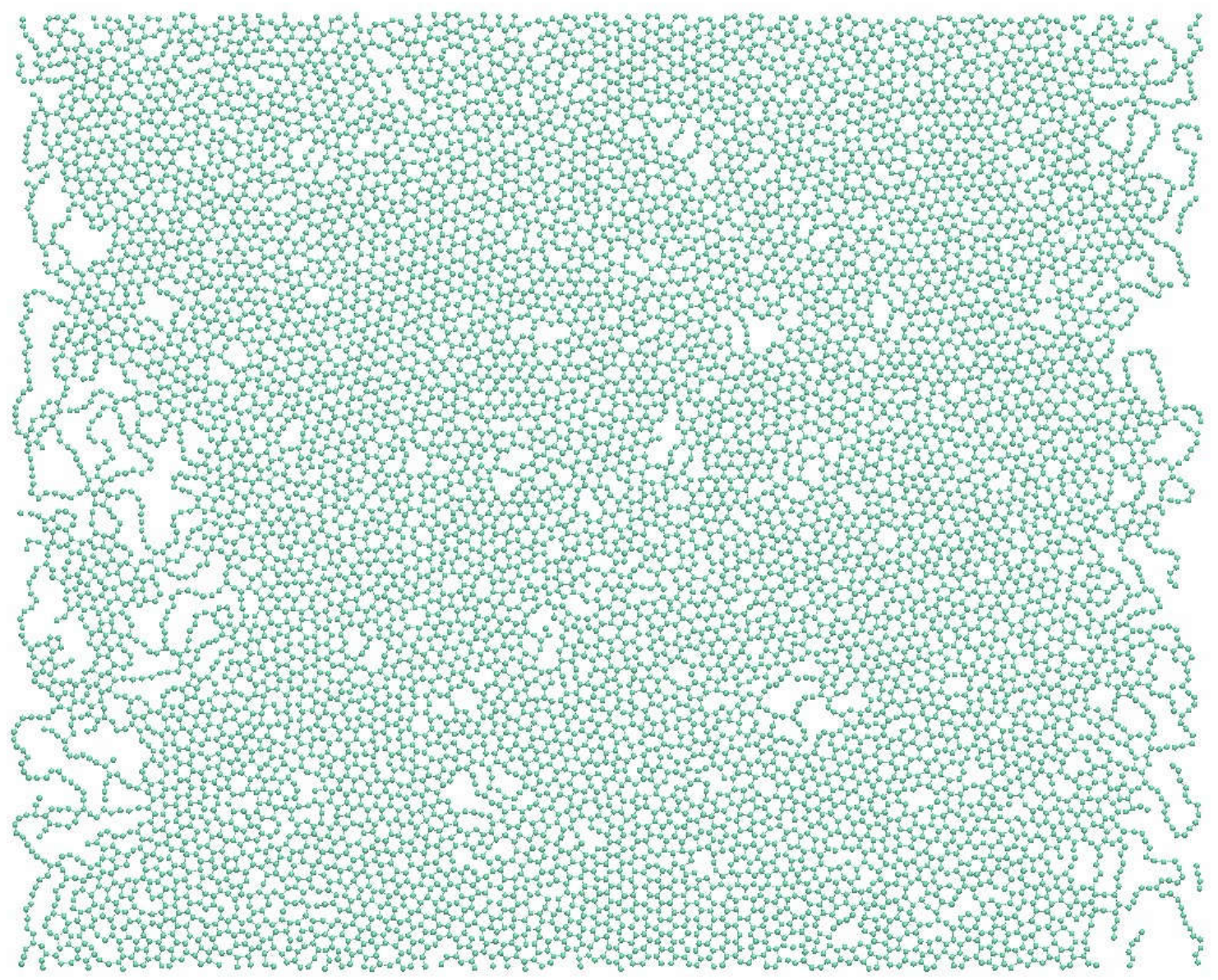

Fig. 6. A side view of $2 \mathrm{D}$ visualization of atomic configurations obtained at $8000 \mathrm{~K}$ for ZGNR.

As is known that the structural defects play an important role in melting process of graphene, the formation of extended defects in graphene from the coalescence of individual mobile vacancies can significantly alter its mechanical, electrical and chemical properties. Various structural defects and their role for graphene have been reviewed [47]. The most popular defects found in graphene can be listed as follows: dislocation 5-7, SW defects, and multiple vacancies including di-vacancies (DVs). Lee et al. carried out tight-binding molecular dynamics simulations using 
model with 120 atoms and they found that at $2800 \mathrm{~K}$ reconstruction process of two hexagons into a pentagon-heptagon occurs at the edge of a ZGNR.

In the present work, for both AGNR and ZGNR with 10000 atoms, the 5-7 dislocations appear at around $2300 \mathrm{~K}$ and that point of temperature can be considered as the pre-melting point. One can say that the nuclei of heating of GNR are the 5-7 dislocations due to their first appearance. Note that, the nuclei of heating of graphene are SW which appear at $2700 \mathrm{~K}$ [13]. Here we present the evolution of defects of ZGNR. We found that the SW and DVs defects are observed contemporaneously at temperature around $2700 \mathrm{~K}$ (Fig. 7). Note that, for graphene, SW defects are found near $3800 \mathrm{~K} \mathrm{[13]} \mathrm{and} \mathrm{DVs} \mathrm{defects} \mathrm{are} \mathrm{observed} \mathrm{at} \mathrm{later} \mathrm{temperature.}$

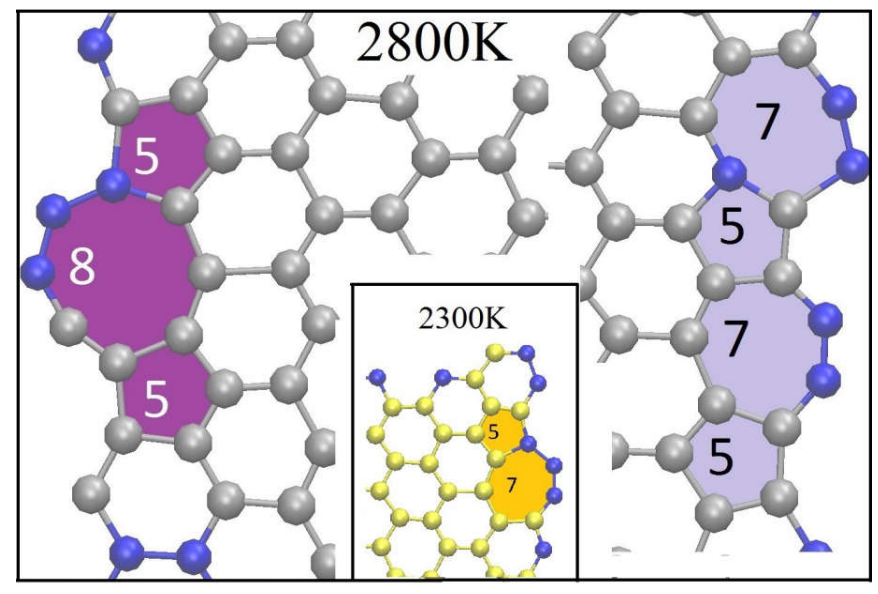

Fig. 7. Defects upon heating of ZGNR: 5-7 dislocations at T=2300 K; SW (5-7-5-7) and DVs $(5-8-5)$ at $T=2800 \mathrm{~K}$.

\section{CONCLUSION}

We have carried out a comprehensive MD simulation of melting process of models with different sizes and edge structures in $2 \mathrm{D}$ case. One can see that in the range of this study, the increasing number of atoms in the model leads to decrease statistical noise and total energy per atom upon heating. Two types of edge structure - AGNR and ZGNR are presented to study the affection of edge structure on melting process. The results show that for both AGNR and ZGNR the phase transition has first order behaviour and the nuclei of heating are 5-7 dislocations which appear at temperature near $2300 \mathrm{~K}$. However, the total energy of AGNR has tendency to shift to the higher region with increasing temperature in comparison with the one of ZGNR. The formation of rings as well as the coordination number of ZGNR and AGNR is different in each range of temperature. In the range of temperature from $300 \mathrm{~K}$ to $6000 \mathrm{~K}$ the formation of rings as well as the coordination number of AGNR is more active than that of ZGNR, after that the one of ZGNR prevails. At temperature $8000 \mathrm{~K}$ both AGNR and ZGNR models still remain solid atoms for indicating that the melting process is not supported by BKTNHY theory. 


\section{ACKNOWLEDGMENTS}

Authors thank for financial support from the National Foundation for Science and Technology Development (NAFOSTED) under grant 103.01.2015.101.

\section{REFERENCES}

[1] K. Novoselov, A.K. Geim, S. Morozov, D. Jiang, M. Katsnelson, I. Grigorieva, S. Dubonos, A. Firsov, Nature, 438 (2005) 197.

[2] Y. Zhang, Z. Jiang, J. Small, M. Purewal, Y.W. Tan, M. Fazlollahi, J. Chudow, J. Jaszczak, H. Stormer, P. Kim, Phys. Rev. Lett., 96 (2006) 136806.

[3] A.K. Geim, K.S. Novoselov, Nature materials, 6 (2007) 183.

[4] I. Meric, M.Y. Han, A.F. Young, B. Ozyilmaz, P. Kim, K.L. Shepard, Nature nanotechnology, 3 (2008) 654.

[5] W.L. Wang, S. Meng, E. Kaxiras, Nano Letters, 8 (2008) 241.

[6] Daniel R. Cooper, Benjamin D’Anjou, Nageswara Ghattamaneni, Benjamin Harack, Michael Hilke, Alexandre Horth, Norberto Majlis, Mathieu Massicotte, Leron Vandsburger, Eric Whiteway, and Victor Yu, ISRN Condensed Matter Physics, 2012 (2012) 501686.

[7] K. Nakada, M. Fujita, G. Dresselhaus, M.S. Dresselhaus, Phys. Rev. B, 54 (1996) 17954.

[8] Y.W. Son, M.L. Cohen, S.G. Louie, Phys. Rev. Lett., 97 (2006) 216803.

[9] M.Y. Han, B. Özyilmaz, Y. Zhang, P. Kim, Phys. Rev. Lett,. 98 (2007) 206805.

[10] L. Tapasztó, G. Dobrik, P. Lambin, L.P. Biró, Nature nanotechnology, 3 (2008) 397.

[11] A. Savvatimskiy, Carbon, 43 (2005) 1115.

[12] F. Colonna, J. Los, A. Fasolino, E. Meijer, Phys. Rev. B, 80 (2009) 134103.

[13] K. Zakharchenko, A. Fasolino, J. Los, M. Katsnelson, Journal of Physics: Condensed Matter, 23 (2011) 202202.

[14] G.D. Lee, C. Wang, E. Yoon, N.M. Hwang, K. Ho, Phys. Rev. B, 81 (2010) 195419.

[15] M.S. Fuhrer, C.N. Lau, A.H. MacDonald, MRS Bulletin, 35 (2010) 289.

[16] M.Z. Bazant, E. Kaxiras, J. Justo, Phys. Rev. B, 56 (1997) 8542.

[17] J.F. Justo, M.Z. Bazant, E. Kaxiras, V. Bulatov, S. Yip, Phys. Rev. B, 58 (1998) 2539.

[18] N. Marks, Phys. Rev. B, 63 (2000) 035401.

[19] F.H. Stillinger, T.A. Weber, Phys. Rev. B, 31 (1985) 5262.

[20] D.W. Brenner, Phys. Rev. B, 42 (1990) 9458.

[21] D.W. Brenner, O.A. Shenderova, J.A. Harrison, S.J. Stuart, B. Ni, S.B. Sinnott, Journal of Physics: Condensed Matter, 14 (2002) 783.

[22] J. Tersoff, Phys. Rev. Lett., 56 (1986) 632.

[23] J. Tersoff, Phys. Rev. Lett., 61 (1988) 2879.

[24] M. Baskes, Phys. Rev. Lett., 59 (1987) 2666.

[25] M. Finnis, J. Sinclair, Philosophical Magazine A, 50 (1984) 45.

[26] I. Oleinik, D. Pettifor, Phys. Rev. B, 59 (1999) 8500.

[27] D. Pettifor, Phys. Rev. Lett., 63 (1989) 2480.

[28] J. Los, A. Fasolino, Phys. Rev. B, 68 (2003) 024107.

[29] J.H. Los, L.M. Ghiringhelli, E.J. Meijer, A. Fasolino, Phys. Rev. B, 72 (2005) 214102.

[30] K. Zakharchenko, M. Katsnelson, A. Fasolino, Phys. Rev. Lett., 102 (2009) 046808.

[31] S. Plimpton, Journal of computational physics, 117 (1995) 1.

[32] S.L. Roux, J. Appl. Cryst., 43 (2010) 181.

[33] W. Humphrey, A. Dalke, K. Schulten, J. Mol. Graphics, 14 (1996) 33.

[34] B.I. Halperin, D.R. Nelson, Phys. Rev. Lett., 41 (1978) 121.

[35] J.M. Kosterlitz, D.J. Thouless, J. Phys. C: Solid State Phys., 6 (1973) 1181.

[36] A.P. Young, Phys. Rev. B, 19 (1979) 1855.

[37] A.Z. Patashinski, R. Orlik, A.C. Mitus, B.A. Grzybowski, and M.A. Ratner, J. Phys. Chem. C, 114 (2010), 20749.

[38] K.J. Strandburg, Rev. Mod. Phys., 60 (1988), 161.

[39] M.A. Glaser and N.A. Clark, Adv. Chem. Phys., 83 (1993), 543. 
[40] A. Patrykiejew, S. Sokolowski, and K. Binder, Surf. Sci. Rep., 37 (2000), 209.

[41] A.Z. Patashinski and M.A. Ratner, J. Chem. Phys., 106 (1997), 7249.

[42] K. Chen, T. Kaplan, and M. Mostoller, Phys. Rev. Lett., 74 (1995), 4019.

[43] K. Bagchi, H.C. Andersen, and W. Swope, Phys. Rev. E, 53 (1996), 3794.

[44] K. Bagchi, H.C. Andersen, and W. Swope, Phys. Rev. Lett., 76 (1996), 255.

[45] J.C. Meyer, C. Kisielowski, R. Erni, M.D. Rossell, M. Crommie, A. Zettl, Nano letters, 8 (2008) 3582.

[46] R. Oeiras, F. Araújo-Moreira, E. Da Silva, Phys. Rev. B, 80 (2009) 073405.

[47] F. Banhart, J. Kotakoski, A.V. Krasheninnikov, ACS Nano, 5 (2011) 26. 